\title{
RESEÑAS DE CONGRESOS Y JORNADAS
}

\section{THE MANY FACES OF TEACHING AND LEARNING MECHANICS}

\author{
Conference on Physics Education. \\ Utrecht 1984
}

Del 20 al 25 de Agosto de 1984 organizado por el GIREP (Groupe International de Recherche sur l'Enseigment de la Physique) en colaboración con el departamento de física de la universidad de Utrecht ha tenido lugar, en el centro de conferencias WOUDSCHOTEN, situado en Zeist, cerca de Utrecht, una conferencia sobre la enseñanza de la Mecánica, con la asistencia de unos ciento ochenta representantes de distintos paises, de los cuales siete éramos espaf̃oles y tres latinoamericanos.

En el acto de apertura la Vice-ministra de Educación y Ciencia de Holanda llamó la atención sobre la importancia de: - que la fisica fuera obligatoria en la escuela secundaria.

- que no se integraran a nivel de secundaria materias como Física, Biología y Química ya que cada una tenia su propia identidad, y además, estaba demostrado, en los intentos de integración que se habian hecho, que a nive! de secundaria no funcionaban.

- que se tuviera en cuenta que la informática era una ciencia nueva que nada tenía que ver con la física ni con las matemáticas y si se enseñaba en secundaria deberia ser con su propia entidad. Otra cuestión distinta es que los computadores pudieran ser un medio auxiliar más en la ensentanza de cualquier materia.

- que lo más importante para que la enseñanza fuera buena es que los profesores estuvieran bien preparados. Un buen profesor podfa hacer mucho incluso sin la colaboración del gobierno.

Como denominador común de un prjmer bloque de ponencias podemos considerar la insistencia en la necesidad de recurrir a ejemplos de la vida diaria para ensefrar cualquier tema de fisica. En este sentido podriamos destacar el proyecto «PLON» de Holanda que presentaron como ejemplo una unidad llama- da "PUENTES» en la que los alumnos comenzaban por visitar puentes de la localidad donde vivían o localidades cercanas, hactan esquemas de ellos buscando los puntos de mayor tensión o presión y la relación de estos puntos con la estructura. A continuación en el Iaboratorio hacian maquetas de estos puentes y comprobaban qué sucedía al someterlos a distintos pesos, y cómo se podían reforzar los puntos débiles. $\mathrm{Se}$ hacia una discusion del fundamento fisico. El proyecto inglés «PHYSICS PLUS» consiste en la producción de una serie de "panfletos", de dos hojas, con ejemplos de la vida diaria para que los profesores los puedan utilizar en sus explicaciones. Un proyecto de la universidad de Kiel consiste en el estudio de temas de la vida diaria aplicando los principios de física; como ejemplo presentaron "la seguridad en el tráfico».

Otro proyecto de la universidad de Frankfurt parte de una serie de experiencias estructuradas, muy sencillas, para hacer el estudio del movimiento e introducir los conceptos de velocidad, aceleración, fuerza... Similar a este es el proyecto de la Escuela Real de Copenhague para alumnos de 14 años.

Con esta misma idea dio una conferencia el Profesor Rogers, de la Universidad de Princenton y director del proyecto Nuffield. Insistió en ia importancia del entusiasmo del profesor por la enseñanza, ilusión que sería capaz de transmitir a sus alumnos. Se podria asegurar que si el profesor amaba la materia el alumno la terminaría amando. Para captar el entusiasmo del alumno y para que el alumno supiera a lo que nos referiamos consideraba muy importantes las experiencias y como ejemplo hizo algunas experiencias sencillas, con material que él mismo se había fabricado. El profesor Rogers, trabaja en la actualidad en la puesta en marcha de un museo de la ciencia en París.

Dos profesores de la Universidad de Kansas presentaron un programa grabado en videodisco sobre el accidente del puente de Tacoma. Recogía parte de una película sobre el funcionamiento del puente y el accidente $y$ por otra parte un estudio de la acción del vien- to sobre un puente colgante: oscilaciones, tensiones, etc. que se presentaban al variar la velocidad del viento y la frecuencia de oscilación. Sobre la misma pantalla varjaban las posibilidades con un microcomputador para ver el efecto. Llamó la atención la claridad de sonido e imagen del videodisco, así como la capacidad de información que puede recoger y lo fácil que es localizarla. El gran inconveniente es el precio.

Con relación al tema de investigación en enseñanza merecen destacar una ponencia de la doctora $R$. Driver de la universidad de Leeds y otra la doctora E. Satiel de París. La primera constituye un análisis de las estructuras conceptuales de los alumnos en mecánica y de las implicaciones en la enseñanza. E. Saltiel estudia las relaciones entre la forma en que los alumnos adquieren los conocimientos y la historia de la física. Se presentaron además numerosas comunicaciones sobre esta línea de investigación centradas en los esquemas conceptuales, y otras sobre uso de microcomputadoras, resolución de problemas, etc. etc. Las ponencias y comunicaciones serán editadas en las correspondientes actas.

Otra actividad fue una visita y posteriormente una conferencia sobre la barrera que están construyendo los holandeses para proteger el delta de las inundaciones en tiempo de tormenta y que consiste en cerrar mediante diques todas las bocas excepto la de Amberes y la de Roterdan.

En cuanto a los programas de computadores nos parecto interesante el del profesor Ogborn de la universidad de Londres que utiliza los mircrocomputadores para que los alumnos, en los tiempos libres, y en grupos pequerios, con unas ideas mínimas del profesor, hagan su propio programa para representar un fenomeno tisico determinado. De esta forma llegarian a entender muy bien el fenómeno porque tendrían que buscar todas las variables que intervienen y cómo intervienen.

Tuvimos también la posibilidad de visitar varios centros. Una escuela de se- 
cundaria que nos llamó la atención por el rigor en la disciplina. Los alumnos de secundaria deben de pasar un examen de prácticas de Fisica antes de ir a la universidad. Un centro de formación de profesores de secundaria en el que los futuros profesores deben elegir dos materias. Una de las opciones más corrientes son física-matemáticas. En física seguían como manual el PSSC. Los estudios duran cuatro affos y en los dos primeros hacen experiencias normales de laboratorio, de nivel de secundaria siguiendo una ficha de trabajo, pero en los dos últimos deben idear ellos sus propias experiencias. El departamento de Fisica de la universidad cu- yos estudios duraban cuatro anos, aunque disponían de uri máximo de seis para completarlos. Seguían libros similares a la universidad de Berkeley y se les exigia un número mínimo de experiencias en el laboratorio para poder pasar. Un Centro de Profesores donde tenian montadas experiencias para que pudieran hacerlas los profesores de secundaria. Tenian por otra parte una serie de experiencias de física nuclear que ellos mismos llevaban a los distintos centros de secundaria. Estaba montado asi porque por razones de seguridad estas experiencias las debia de hacer un experto. Como el pais tiene distancias muy pequentas no es problema el desplaza- miento ni de los profesores ni del personal del centro

Se acordó que la próxima reunión del GIREP fuera en Dinamarca sobre ASTRONOMIA y se eligió como presidente del GIREP para el próximo mandato, en sustitución del Profesor Lo ria de Modena (Italia), al Profesor Black de Chelsea College de Londres (Inglaterra).

$M^{a}$ TERESA MARTIN SANCHEZ Centro de Ensexanzas Integradas Zamora

MANUELA MARTIN SANCHEZ E.U. "Pueblo Montesino» Madrid 